University of Nebraska - Lincoln

DigitalCommons@University of Nebraska - Lincoln

Timothy J. Gay Publications

Research Papers in Physics and Astronomy

2009

\title{
Spin Torque on Molecular Rotation Induced by Polarized Electrons
}

\author{
J. W. Maseberg \\ Behlen Laboratory of Physics, University of Nebraska, Lincoln, Nebraska
}

Timothy J. Gay

University of Nebraska - Lincoln, tgay1@unl.edu

Follow this and additional works at: https://digitalcommons.unl.edu/physicsgay

Part of the Physics Commons

Maseberg, J. W. and Gay, Timothy J., "Spin Torque on Molecular Rotation Induced by Polarized Electrons" (2009). Timothy J. Gay Publications. 50.

https://digitalcommons.unl.edu/physicsgay/50

This Article is brought to you for free and open access by the Research Papers in Physics and Astronomy at DigitalCommons@University of Nebraska - Lincoln. It has been accepted for inclusion in Timothy J. Gay Publications by an authorized administrator of DigitalCommons@University of Nebraska - Lincoln. 


\title{
Spin torque on molecular rotation induced by polarized electrons
}

\author{
J. W. Maseberg * and T. J. Gay \\ Behlen Laboratory of Physics, University of Nebraska, Lincoln, Nebraska 68588-0111, USA
}

(Received 15 October 2008; published 5 February 2009)

\begin{abstract}
We have measured the linear and circular polarization of $391.4 \mathrm{~nm} \mathrm{~N}{ }_{2}^{+} B^{2} \Sigma_{u}^{+}\left(v^{\prime}=0\right) \rightarrow X^{2} \Sigma_{g}^{+}\left(v^{\prime \prime}=0\right)$ $P$-branch fluorescence produced by spin-polarized electron impact excitation of $\mathrm{N}_{2} X^{1} \Sigma_{g}^{+}(v=0)$ ground states. The resulting nonzero values of the circular polarization indicate that spin-rotation coupling, in conjunction with electron exchange excitation, acts to produce oriented rotational angular momenta in the excited molecular state prior to decay.
\end{abstract}

DOI: $10.1103 /$ PhysRevA.79.022705

\section{INTRODUCTION}

Use of spin-polarized electrons in collisions with atoms and molecules provides a direct probe of spin-dependent collision dynamics $[1,2]$. A prototypical example of this is the production of circularly polarized fluorescence resulting from the transfer of spin angular momentum to orbital orientation in a collisions-excited atomic target [3]. In such reactions, the target becomes spin polarized by an exchange collision with a polarized electron beam. For the fluorescence to be circularly polarized, there must exist a mechanism to couple the newly oriented spin angular momentum, $S$, with the initially unoriented electron orbital angular momentum $\boldsymbol{L}$. This is accomplished by the spin-orbit interaction, in which $\boldsymbol{L}$ and $\boldsymbol{S}$ couple to form $\boldsymbol{J}$, which remains fixed in space (ignoring nuclear spin). Due to the precession of $\boldsymbol{L}$ and $\boldsymbol{S}$ about $\boldsymbol{J}, \boldsymbol{L}$ becomes spatially oriented and circular polarization (relative Stokes parameter $P_{3} \neq 0$ ) can be observed, provided that the time scale of the $\boldsymbol{L} \cdot \boldsymbol{S}$ coupling is less than or comparable to the excited state lifetime.

Molecular orientation can be considered in a similar manner. Recently, circular polarization of the fluorescence from excited $\mathrm{H}_{2}$ triplet molecular states produced by spinpolarized electron impact has been observed [4]. (The identification of triplet states ensures that spin exchange has occurred.) Values of circular polarization normalized to the spin polarization of the electron beam, $P_{3} / P_{e}$, of $\sim 0.1$ were observed for a mixture of Fulcher band transitions in the 595-605 nm wavelength region. The mechanism for this polarization production is similar to that in atoms, but with an added layer of complication due to the molecular angular momentum coupling scheme (see Fig. 1). In Hund's case (b) molecules such as $\mathrm{H}_{2}$, the projection of the electronic orbital angular momentum onto the internuclear axis $(\boldsymbol{\Lambda})$ couples with the nuclear rotational angular momentum $(\boldsymbol{N})$ to form the total orbital angular momentum $(\boldsymbol{K})$, which in turn couples with the electron spin $(\boldsymbol{S})$ to form the total angular momentum $(\boldsymbol{J})$. (Note that $N$ is not a good quantum number unless $\Lambda=0$ so that $K=N$.) Circular polarization is produced when $\boldsymbol{K}$ is oriented by virtue of its coupling with $S$ through a spin-orbit term in the molecular Hamiltonian $A(\boldsymbol{S} \cdot \boldsymbol{K})$ $=A \boldsymbol{S} \cdot(\boldsymbol{\Lambda}+\boldsymbol{N})$. As in atoms, this occurs over the spincoupling time, i.e., the inverse precession frequency of $S$

*maseberg@bigred.unl.edu
PACS number(s): $34.80 . \mathrm{Nz}, 33.15 . \mathrm{Pw}, 34.80 . \mathrm{Gs}$

about $\boldsymbol{J}$. Such times are comparable to those in the constituent atomic cases. In the experiments of Green et al. [4], the fluorescence was primarily from $\Pi$ states $(\Lambda=1)$, and the ultimate molecular orientation was mainly due to the electron spin-orbit interaction with $\boldsymbol{\Lambda}$ (as opposed to $N$ ). This is because the magnetic field in the rest frame of the electrons due to their orbital motion is much larger than that due to the (relatively slow) rotation of the nuclei themselves.

In the experiment reported here, we demonstrate that the collision-induced orientation of $\boldsymbol{K}$, and hence $P_{3}$ production, is possible even in the absence of electronic orbital angular momentum along the internuclear axis $(\Lambda=0$ and $\boldsymbol{K}=\boldsymbol{N})$. The mechanisms responsible for this torque on the molecular nuclei are the direct coupling of $S$ to the magnetic field produced by the "current loop" of nuclear rotation, as well as the coupling of $S$ to the residual electronic orbital angular momentum perpendicular to the internuclear axis, which also produces a magnetic field along $\boldsymbol{K}$ [5-7]. [The latter mechanism can also be attributed to nuclear rotation, however, in that it requires uncoupling of the total electron orbital angular momentum $(\boldsymbol{L})$ from the internuclear axis.] To date, the

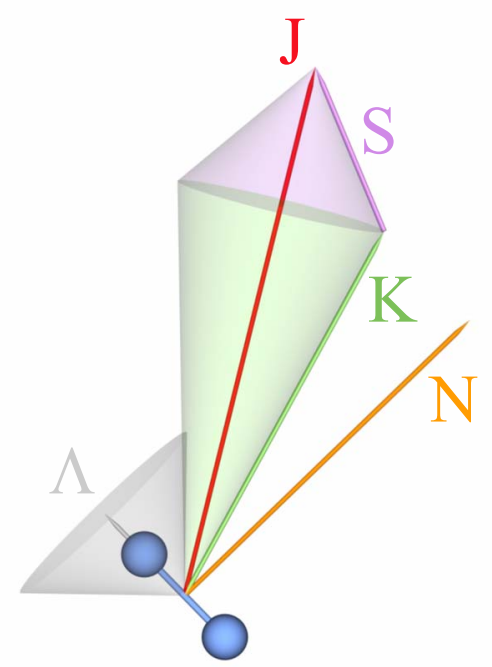

FIG. 1. (Color online) Schematic of Hund's case (b) coupling for a $\Lambda=1(\Pi)$ state. The $S$ and $\boldsymbol{K}$ vectors precess around the total angular momentum $\boldsymbol{J}$ in the fine-structure coupling time. Initial orientation of $\boldsymbol{S}$ leads to orientation of $\boldsymbol{J}$ and $\boldsymbol{K}$. The vector $\boldsymbol{N}$ is always perpendicular to the internuclear axis, and the precession loci of $\boldsymbol{L}$, $\boldsymbol{K}$, and $\boldsymbol{S}$ are indicated by transparent cones. 


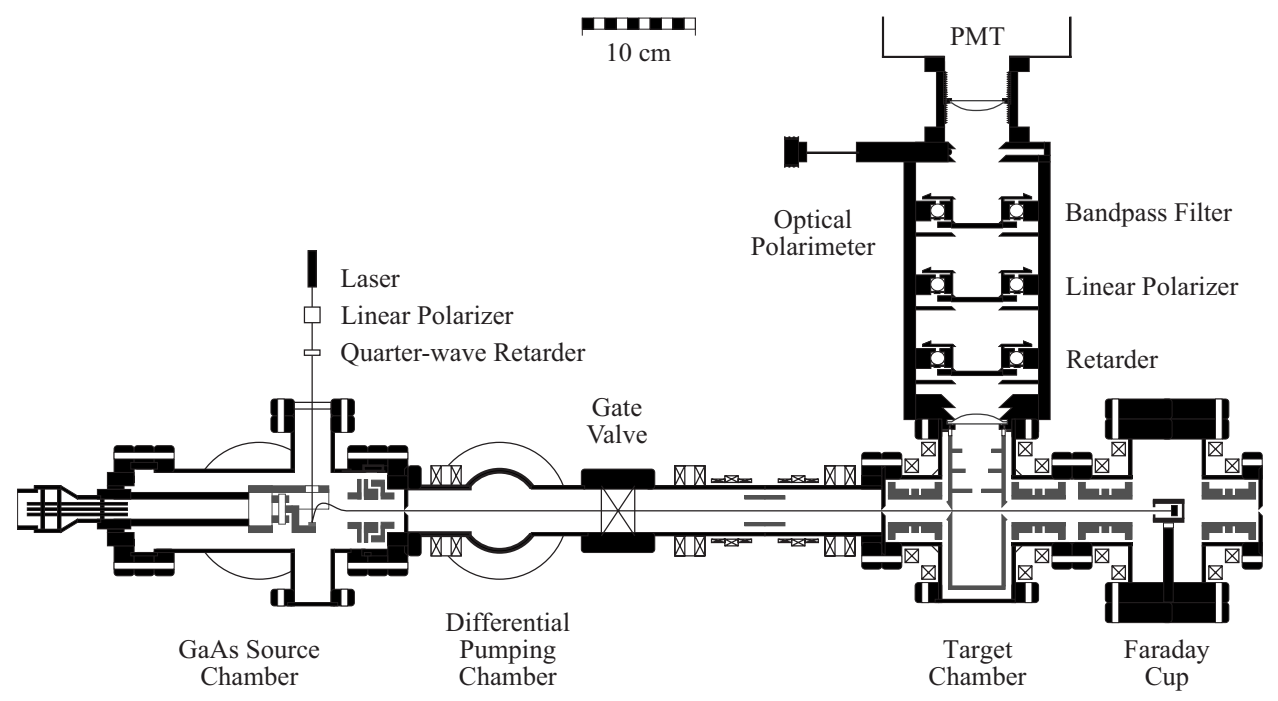

FIG. 2. Experimental apparatus.

effects of these rotational couplings have only been observed as "spin doubling" in molecular spectra [8], although it has been discussed as a possible mechanism for spin relaxation in triplet alkali dimers $[9,10]$. For ${ }^{2} \Sigma$ states, this so-called "spin-rotation" term in the Hamiltonian, $\gamma(\boldsymbol{K} \cdot \boldsymbol{S})$, gives rise to energy splittings

$$
\Delta E_{\mathrm{SR}} \simeq \gamma\left(K+\frac{1}{2}\right) .
$$

Our experiment provides direct evidence for molecular orientation due to spin-rotation coupling in electron-molecule collisions.

\section{EXPERIMENT}

We have studied the excitation-ionization reaction with $\mathrm{N}_{2}$ excited by spin-polarized electrons leading to fluorescence in the first negative system of $\mathrm{N}_{2}{ }^{+}$,

$$
\begin{aligned}
e+\mathrm{N}_{2} X^{1} \Sigma_{g}^{+}(v=0) & \rightarrow \mathrm{N}_{2}^{+} B^{2} \Sigma_{u}^{+}\left(v^{\prime}=0\right)+2 e \\
& \rightarrow \mathrm{N}_{2}{ }^{+} X^{2} \Sigma_{g}^{+}\left(v^{\prime \prime}=0\right)+2 e+\gamma(391 \mathrm{~nm}) .
\end{aligned}
$$

Our experimental apparatus is shown in Fig. 2. To obtain a beam of spin-polarized electrons, circularly polarized $785 \mathrm{~nm}$ light from a laser diode irradiates an unstrained $\mathrm{GaAs}$ crystal. Layers of cesium and oxygen are applied to the crystal in order to produce a negative electron affinity surface, allowing electrons to escape [11] with a typical yield of $1-10 \mu \mathrm{A} / \mathrm{mW}$ and an energy width $<0.4 \mathrm{eV}$. The crystal is held at a potential of $-250 \mathrm{~V}$ to define the beam transport energy. Photoelectrons are electrostatically deflected by $90^{\circ}$ so that the spin polarization becomes transverse with respect to the beam direction. The spin direction is determined by rotating the quarter-wave retarder controlling the laser polarization. The amount of spin-polarization, $P_{e}$, is $27 \%$, as measured by optical electron polarimetry [12]. A differential pumping region separates the field-free target gas cell from the source. The target potential is varied to change the interaction energy. Upon exiting the target, the electron beam is collected in a Faraday cup for current normalization. A lens above the target cell images light from excited molecules into an optical polarimeter [13], comprising a rotatable quarter-wave retarder, linear polarizer, bandpass filter and Hamamatsu R946 photomultiplier tube.

Data acquisition consisted of incrementing the beam energy every $5 \mathrm{~s}$; for each energy interval, the integrated counts, average current, and average pressure were measured. After a single energy scan was completed, the beam energy was reset to the starting value and the angle of the retarder in the optical polarimeter incremented by $22.5^{\circ}$. This process continued until a full rotation of the polarimeter retarder was achieved. The angle of the laser quarter-wave retarder was then incremented by $90^{\circ}$, changing the spin direction of the electrons to help minimize instrumental asymmetries. A single "run" comprised several full rotations of the laser retarder.

Below-threshold unnormalized count rates were averaged to determine the background, which was then subtracted from all count rates. (Chauvenet's criterion was applied to remove anomalous count rates [14].) Above-threshold count rates were then normalized to pressure and current, and count rates corresponding to unique energies and orientations of the optical retarders were combined to give final intensities and their uncertainties. For a given energy, the intensities for different optical orientations were subjected to an inverse Fourier transform to obtain Fourier coefficients related to the desired Stokes parameters [15].

The polarization of molecular fluorescence depends on the rotational transitions observed. To illustrate our optical sensitivity, Fig. 3 shows a spectrum for the $\mathrm{N}_{2}^{+} B{ }^{2} \Sigma_{u}^{+}\left(v^{\prime}\right.$ $=0) \rightarrow X^{2} \Sigma_{g}^{+}\left(v^{\prime \prime}=0\right)$ band excited by electron bombardment [16]. As expected, there is a 2:1 intensity alternation of even $K^{\prime \prime}$ vs odd $K^{\prime \prime}$ rotational transitions due to the nuclear spin statistics of ${ }^{14} \mathrm{~N}_{2}$ [8]. The effect of the narrow bandpass filter [center wavelength $391.6 \mathrm{~nm}$; full width at half maximum (FWHM) $0.92 \mathrm{~nm}$ ] used for our experiment is shown in the lower plot, indicating that our data is due only to $P$-branch $\left(K^{\prime}-K^{\prime \prime}=-1\right)$ fluorescence, the $R$ branch $\left(K^{\prime}-K^{\prime \prime}=+1\right)$ being almost completely suppressed. Also, we preferentially 


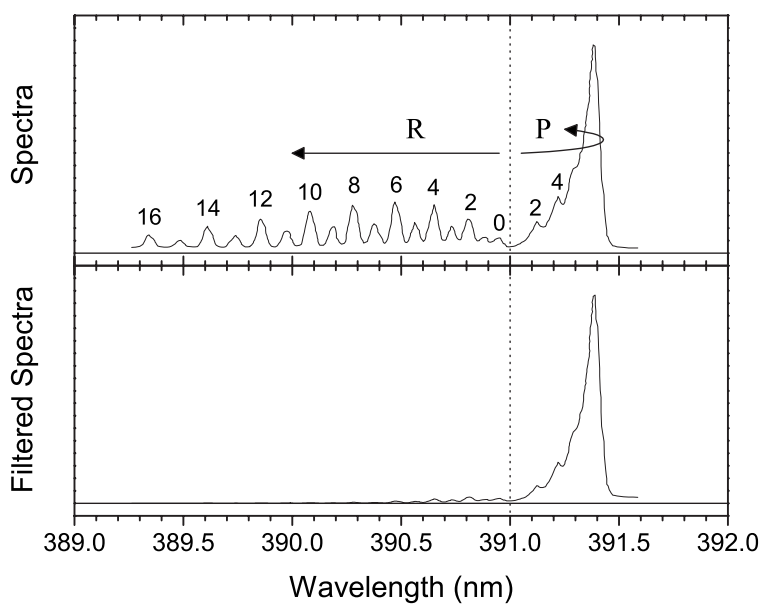

FIG. 3. Upper figure, rotational spectrum for the $391.4 \mathrm{~nm}$ band taken from Ref. [16]; some final rotational states for the $P\left(K^{\prime \prime}\right)$ and $R\left(K^{\prime \prime}\right)$ branches are labeled. Lower figure, spectrum modified by our bandpass filter transmission.

detect transitions near the band head $P\left(K^{\prime \prime} \sim 14\right)$. No significant contaminant molecular or atomic lines are expected to coexist in the profile of the bandpass filter. A transition in the $\mathrm{N}_{2}$ Goldstein-Kaplan $C^{\prime}{ }^{3} \Pi_{u}\left(v^{\prime}=0\right) \rightarrow B{ }^{3} \Pi_{g}\left(v^{\prime \prime}=8\right)$ system at $391.5 \mathrm{~nm}$ exists, but it is apparently much weaker than the transition under study, as we observe negligible intensity from this band below the threshold for $B^{2} \Sigma_{u}^{+}$state production.

\section{RESULTS}

Our linear and circular polarization data presented in Fig. 4 were acquired at a pressure of $4 \times 10^{-2} \mathrm{~Pa}$ to avoid potential effects of radiation trapping. Cascade contributions from higher-lying states are not expected [17]. Our $P_{1}$ values are consistent with those of Ref. [18], but we note that the interference filter used in that work transmitted both the $P$ - and most of the $R$-branch fluorescence. It is difficult to assess what the kinematically required threshold value of $P_{1}$ should be. For production of the $B^{2} \Sigma$ state, preferential excitation of $M_{J}= \pm \frac{1}{2}$ levels is expected [19]. This would yield positive $P_{1}$ values for the thermal ensemble of rotational states present in a room-temperature target, which we do observe. In addition, the effects of hyperfine and fine-structure depolarization are significant [20], leading to an overall reduction in the degree of linear polarization at all energies. The measured values of $P_{2}$ (the $45^{\circ} / 135^{\circ}$ linear polarization fraction), are consistent with zero. This is expected, as the total spin of the collision system should be a good quantum number due to the low $Z$ of the target nuclei [21].

Since the molecular states of interest are all of $\Sigma$ character, the angular momentum coupling is described by Hund's case (b). Figure 5 depicts this coupling scheme, wherein the vectors $\boldsymbol{K}$ and $\boldsymbol{S}$ couple to form the spatially fixed $\boldsymbol{J}$ (total angular momentum excluding nuclear spin). Vectors $\boldsymbol{K}$ and $\boldsymbol{S}$ precess about the spatially fixed $\boldsymbol{J}$ with a period of $<0.1 \mathrm{~ns}$ $(K=1)$ corresponding to the $\gamma(\boldsymbol{K} \cdot \boldsymbol{S})$ "spin-doubling" term in the molecular Hamiltonian [22]. This spin-rotation coupling

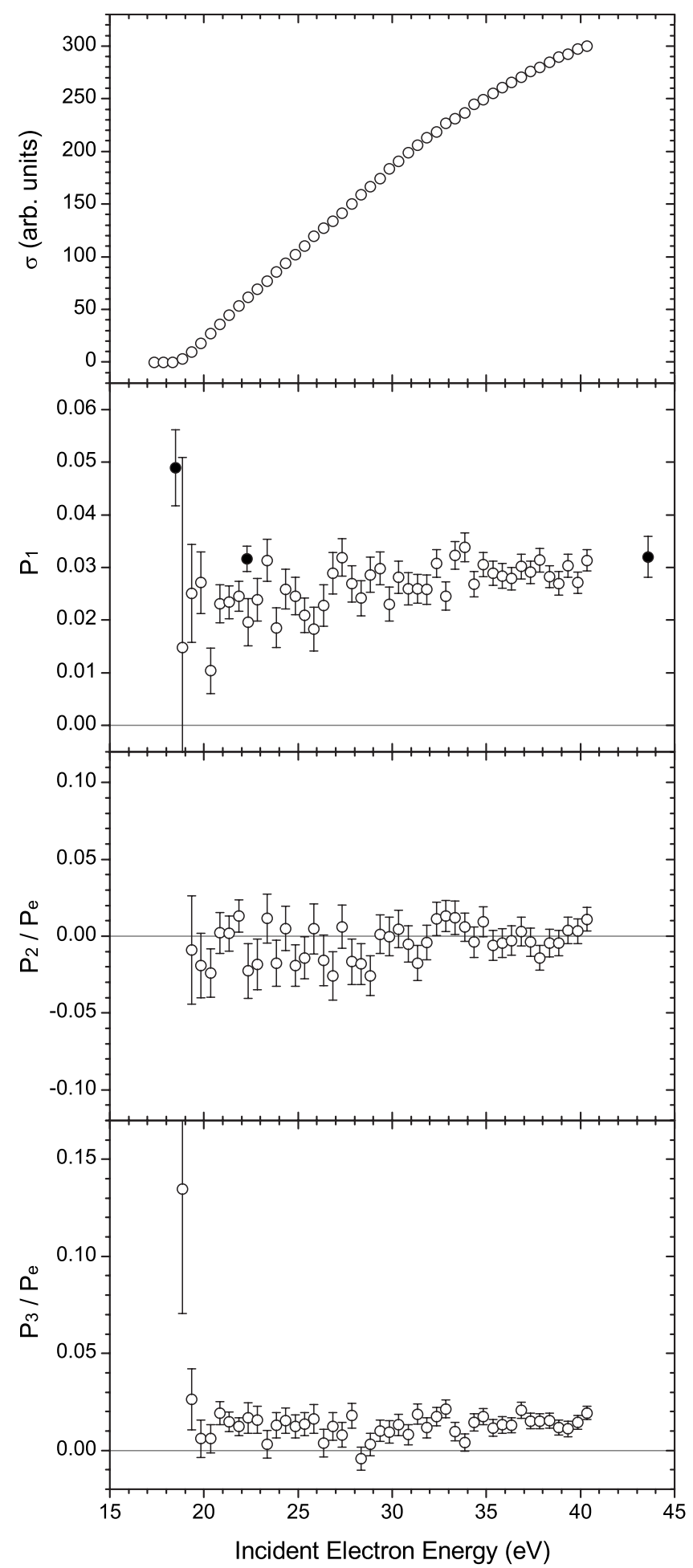

FIG. 4. Relative optical emission cross section and fluorescence polarizations vs electron energy for the $391.4 \mathrm{~nm} \mathrm{~N}{ }_{2}^{+} B^{2} \Sigma_{u}^{+}\left(v^{\prime}\right.$ $=0) \rightarrow X^{2} \Sigma_{g}^{+}\left(v^{\prime \prime}=0\right)$ band. Data of this work is represented by open circles ( $1 \sigma$ errors); data of Ref. [18] by filled circles (errors represent $98 \%$ confidence level). Threshold is at $18.75 \mathrm{eV}$ [23].

time scale is much less than the lifetime of the $B^{2} \Sigma_{u}^{+}$system (61 ns [24]), so time-averaged orientation of $\boldsymbol{K}$ can develop.

The nonzero value of $P_{3} / P_{e}$ for the $391.4 \mathrm{~nm}$ fluorescence provides direct evidence of molecular orientation due 


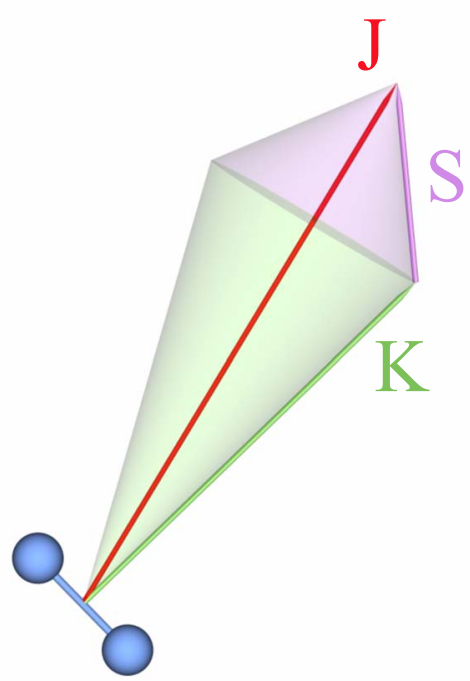

FIG. 5. (Color online) Schematic of Hund's case (b) coupling for a $\Lambda=0(\Sigma)$ state. The $\boldsymbol{S}$ and $\boldsymbol{K}$ vectors precess around the total angular momentum $\boldsymbol{J}$. Initial orientation of $\boldsymbol{S}$ leads to orientation of $\boldsymbol{J}$ and $\boldsymbol{K}$. The vector $\boldsymbol{K}$ is always perpendicular to the internuclear axis.

to this coupling. Fitting a constant to the data gives an energy-averaged value of $0.0133(8)$, with a reduced $\chi^{2}$ $=1.04$. However, the observed values are relatively small when compared to the circular polarizations detected for atomic and even molecular $\mathrm{H}_{2}$ triplet-state fluorescence. There are several reasons that may explain the small values of circular polarization we observe. The first is related to the fact that the $B$ state is a doublet, so electron exchange is not guaranteed, and the initial spin orientation may be diluted relative to a triplet state by an exchange-to-direct excitation cross-section ratio. Another factor to consider is the relative magnitude of rotational state angular momenta in $\mathrm{N}_{2}$ vs $\mathrm{H}_{2}$. For larger values of $K$, spin orientation is less effective at producing appreciable nuclear orbital orientation, resulting in smaller $P_{3}$ values. Room-temperature rotational states in nitrogen are populated up to $K \sim 20$, while for hydrogen the maximum $K$ is $\sim 3$. Thus the doublet state spin momentum of $\frac{1}{2} \hbar$ is small compared with the rotational angular momen- tum of $\sqrt{K(K+1)} \hbar$. In fact, this ratio $\left(\sim \frac{1}{40}\right.$ for maximum $\left.K\right)$ is roughly an order of magnitude less than that for triplet state $\mathrm{H}_{2}\left(\sim \frac{1}{3}\right.$ for maximum $\left.K\right)$. This effect alone could account for the differences in the observed $P_{3} / P_{e}$ from these two molecular species. Finally, the results are susceptible to both fine and hyperfine depolarization [20,25]. Typical hyperfine splittings in the excited states of atomic nitrogen are of the order of $10^{8} \mathrm{~Hz}$ [26], so molecular depolarization should occur in $\sim 10 \mathrm{~ns}$.

In this regard, we mention an earlier experiment performed by the Münster group in which the circular polarization for the $\mathrm{N}_{2} C^{3} \Pi_{u}\left(v^{\prime}=0\right) \rightarrow B{ }^{3} \Pi_{g}\left(v^{\prime \prime}=0\right) 337.1 \mathrm{~nm}$ band (second positive system) excited by spin-polarized electrons was measured. Values of $P_{3} / P_{e}$ were found to be zero within an uncertainty of $2 \times 10^{-3}$ [27]. This result is surprising, given that the fully oriented (triplet state) electrons couple strongly to the internuclear axis [Hunds case (a)] and should provide orientation of $\boldsymbol{K}$. We have since confirmed this null result for the $\mathrm{N}_{2} C^{3} \Pi_{u}\left(v^{\prime}=0\right) \rightarrow B{ }^{3} \Pi_{g}\left(v^{\prime \prime}=2\right) 380.5 \mathrm{~nm}$ band. We speculate that it is due to the fact that in both experiments there was no resolution of the rotational band structure of the upper state, giving both $P$ - and $R$-band fluorescence equal weight. This would tend to cause the integrated value of $P_{3}$ to average to zero [28]. Further rotationally isolated measurements are desirable in order to confirm this conjecture.

\section{CONCLUSION}

In conclusion, we have shown that orientation of molecular states can occur via spin exchange even in the absence of direct spin-electron orbit coupling. This results from the magnetic coupling of the electron spin with both nuclear rotation directly, and with its own residual orbital angular momentum perpendicular to the internuclear axis. This experiment illustrates the usefulness of spin-polarized electrons as a probe of molecular collision dynamics.

\section{ACKNOWLEDGMENTS}

This work was supported by NSF Grant No. PHY0653379. The authors wish to thank Gordon Gallup and Friedrich Hanne for useful discussions.
[1] J. Keßler, Polarized Electrons, 2nd ed. (Springer-Verlag, Berlin, 1985).

[2] N. Andersen and K. Bartschat, Polarization, Alignment, and Orientation in Atomic Collisions (Springer-Verlag, New York, 2001).

[3] J. Goeke, J. Keßler, and G. F. Hanne, Phys. Rev. Lett. 59, 1413 (1987).

[4] A. S. Green, G. A. Gallup, M. A. Rosenberry, and T. J. Gay, Phys. Rev. Lett. 92, 093201 (2004).

[5] C. H. Townes and A. L. Schawlow, Microwave Spectroscopy (Dover, New York, 1975).

[6] J. H. Van Vleck, Phys. Rev. 33, 467 (1929).
[7] S. Green and R. N. Zare, J. Mol. Spectrosc. 64, 217 (1977).

[8] G. Herzberg, Molecular Spectra and Molecular Structure I. Spectra of Diatomic Molecules, 2nd ed. (D. Van Nostrand Company, Princeton, NJ, 1950).

[9] C. J. Erickson, D. Levron, W. Happer, S. Kadlecek, B. Chann, L. W. Anderson, and T. G. Walker, Phys. Rev. Lett. 85, 4237 (2000).

[10] R. A. Bernheim, J. Chem. Phys. 36, 135 (1962).

[11] D. T. Pierce, R. J. Celotta, G.-C. Wang, W. N. Unertl, A. Galejs, C. E. Kuyatt, and S. R. Mielczarek, Rev. Sci. Instrum. 51, 478 (1980).

[12] T. J. Gay, J. E. Furst, K. W. Trantham, and W. M. K. P. Wija- 
yaratna, Phys. Rev. A 53, 1623 (1996).

[13] J. W. Maseberg and T. J. Gay, J. Phys. B 39, 4861 (2006).

[14] P. R. Bevington, Data Reduction and Error Analysis, 3rd ed. (McGraw-Hill, Boston, 2003).

[15] H. G. Berry, G. Gabrielse, and A. E. Livingston, Appl. Opt. 16, 3200 (1977).

[16] W. W. Hunter, Jr., Ph. D. thesis, The College of William and Mary in Virginia, 1965; NASA Report No. NASA-TM-X66993.

[17] J. T. Fons, J. S. Allen, R. S. Schappe, and C. C. Lin, Phys. Rev. A 49, 927 (1994).

[18] D. H. Crandall, W. E. Kauppila, R. A. Phaneuf, P. O. Taylor, and G. H. Dunn, Phys. Rev. A 9, 2545 (1974).

[19] I. C. Malcolm, H. W. Dassen, and J. W. McConkey, J. Phys. B 12, 1003 (1979).

[20] J. A. Guest, K. H. Jackson, and R. N. Zare, Phys. Rev. A 28, 2217 (1983).
[21] J. E. Furst, T. J. Gay, W. M. K. P. Wijayaratna, K. Bartschat, H. Geesmann, M. A. Khakoo, and D. H. Madison, J. Phys. B 25, 1089 (1992).

[22] E. A. Colbourn and A. E. Douglas, J. Mol. Spectrosc. 65, 332 (1977).

[23] Wm. B. Peatman, B. Gotchev, P. Gürtler, E. E. Koch, and V. Saile, J. Chem. Phys. 69, 2089 (1978).

[24] H. Schmoranzer, P. Hartmetz, D. Marger, and J. Dudda, J. Phys. B 22, 1761 (1989).

[25] K. Blum and H. Jackubowicz, J. Phys. B 11, 909 (1978).

[26] P. Cangiano, M. de Angelis, L. Gianfrani, G. Pesce, and A. Sasso, Phys. Rev. A 50, 1082 (1994).

[27] G. F. Hanne, in Novel Aspects of Electron-Molecule Collisions, edited by K. Becker (World Scientific, Singapore, 1998); C. Mette et al., Verhandl. DPG 29, 462 (1994).

[28] R. N. Zare, Angular Momentum (Wiley, New York, 1988). 